\title{
Caracterização de uso e cobertura da terra na Amazônia utilizando imagens duais multitemporais do COSMO-SkyMed
}

\author{
Adriana Rodrigues de AZEVEDO ${ }^{1 *}$, João Roberto dos SANTOS², Fábio Furlan GAMA², Paulo Maurício Lima \\ de Alencastro GRAÇA³ ${ }^{3}$ José Cláudio MURA² \\ 1 Instituto Chico Mendes de Conservação da Biodiversidade - ICMBio. EQSW 103/104, Complexo Administrativo, Sudoeste, CEP: 70.670-350. Brasília, DF, Brasil. \\ * Autor Correspondente: adriana.azevedo@icmbio.gov.br \\ 2 Instituto Nacional de Pesquisas Espaciais, Coordenação de Observação da Terra - OBT/INPE. Av. dos Astronautas, 1758, CEP: 12.227-010. São José dos Campos, São Paulo, \\ Brasil. jroberto@dsr.inpe.br, fabio@dpi.inpe.br, mura@dpi.inpe.br. \\ ${ }^{3}$ Instituto Nacional de Pesquisas da Amazônia, Coordenadoria de Dinâmica Ambiental - CDAM/INPA. Av. André Araújo, 2936, CEP: 69060-001. Manaus, Amazonas, Brasil. \\ pmlag@inpa.gov.br.
}

\section{RESUMO}

A utilização de imagens de radar é fonte alternativa de informaçóes para subsidiar o monitoramento da região amazônica, visto que as imagens ópticas têm limitaçóes de imageamento em zonas tropicais face a ocorrência de nuvens. Por conseguinte este trabalho teve como objetivo analisar a capacidade das imagens-radar de banda X multitemporais e polarizadas obtidas pelo satélite COSMO-SkyMed (COnstellation of small Satellites for Mediterranean basin Observation), no modo intensidade, isoladamente e agregados às informaçóes texturais, na caracterização temática de uso e cobertura da terra no município de Humaitá/AM. A metodologia empregada consistiu da: análise das imagens duais obtidas em duas aquisiçóes subsequentes, de forma a explorar a potencialidade do conjunto de dados na forma quad-pol intensidade; extração dos atributos texturais a partir da matriz de coocorrência (Gray Level Co-occurrence Matrix) e posterior classificação contextual; avaliação estatística de desempenho temático das imagens intensidade e texturais, isoladas e em grupos polarizados. Dentre os vários resultados alcançados, foi verificado que o grupo formado somente pelas imagens intensidade apresentou o melhor desempenho, comparado àqueles contendo os atributos texturais. Nesta separabilidade, estavam envolvidas as classes de floresta, floresta aluvial, reflorestamento, savana, pasto e queimada, obtendo-se 66\% de acurácia total e valor Kappa de 0,55. Os resultados mostraram que as imagens de banda X do COSMO-SkyMed, modo StripMap (Ping-Pong), multipolarizadas, têm potencial moderado para a caracterização e monitoramento da dinâmica de uso e cobertura da terra na Amazônia brasileira.

PALAVRAS-CHAVE: radar, mapeamento florestal, análise textural, monitoramento.

\section{Land use/land cover characterization in Amazonia using COSMO-SkyMed multitemporal images}

\section{ABSTRACT}

The use of radar imagery is an alternative source of information to support the monitoring of the Amazon region, since the optical images have imaging limitations in tropical areas due to the occurrence of clouds. Therefore, the goal of this study is to analyze the radar images in X-band multi-temporal polarized obtained by COSMO-SkyMed satellite (COnstellation of small Satellites for Mediterranean basin Observation), in the intensity mode, isolated and/or combined with textural information, to thematic characterization of land use/land cover in the Humaitá, Amazonas State region. The methodology used includes: analysis of the dual images obtained during two subsequent acquisitions, in order to explore the potential of the dataset as a quad-pol intensity; extraction of textural attributes from the co-occurrence matrix (Gray Level Co-occurrence Matrix) and subsequent contextual classification; statistical assessment of the thematic performance of the intensity and textural images, isolated and in polarized groups. Within the results achieved, the group formed only by the intensity images presented a better performance if compared to those containing the textural attributes. In this discrimination, the classes involved were forest, alluvial forest, reforestation, savannah, pasture and burned areas, yielding 66\% overall accuracy and a Kappa value of 0.55 . The results showed that X band images, from COSMO-SkyMed, StripMap mode (Ping-Pong), multi-polarized, presents a moderate potential to characterize and monitor the dynamics of land use/land land cover in the Brazilian Amazon.

KEYWORDS: radar, forest mapping, textural analysis, monitoring. 


\section{INTRODUÇÃO}

O monitoramento da expansão da atividade agrícola e pecuária na regiáo Amazônica tem sido realizado extensamente por meio de sensores remotos ópticos, auxiliando no controle e fiscalização do desmatamento (Graça et al. 2007). Porém, o uso de tais ferramentas é prejudicado pelas condiçóes de intensa cobertura de nuvem, comuns nessa regiáo tropical (Asner 2001). Dessa forma, o emprego das imagens de radar de abertura sintética (SAR) tem se mostrando uma ferramenta útil para o mapeamento, subsidiando nas tarefas de controle e fiscalização de processos antrópicos na paisagem florestal, por permitir a aquisição de dados independente da iluminaçấo solar e das condiçôes climáticas, além de fornecer informaçôes sobre textura, propriedades dielétricas e conteúdo de umidade presente no alvo (Balzter 2001).

Os sistemas SAR vêm sendo utilizados em florestas tropicais para mapeamento de estrutura florestal e de uso e cobertura do solo (Hoekman e Quiñones 2000; Gonçalves et al. 2011; Le Toan et al. 2011; Saatchi et al. 2011). Para identificaçáo das diferentes fitofisionomias em regiôes tropicais, estudos mostram que as bandas $\mathrm{P}$ e L, nas polarizaçôes cruzadas (HV onde o sinal-radar é transmitido na polarização Horizontal e recebido na Vertical; e VH sendo transmitido na polarização Vertical e recebido na Horizontal), têm apresentado significativo potencial (Kasischke et al. 1997; Yanasse et al. 1997; Santos et al. 2003). Por sua vez, Garcia et al. (2012) mostraram que a utilizaçáo das imagens TerraSAR-X (banda $\mathrm{X}$ ), através da combinação de imagens amplitude das componentes $\mathrm{HH}$ (radiação transmitida e recebida na polarização Horizontal) e VV (radiação transmitida e recebida na polarização Vertical), da informação de fase $\left(H H . V V^{*}\right)$ e de entropia, formando o conjunto $\left(A_{H H}, A_{v v}, A_{<H H . V^{*}>}, A_{\text {entropia }}\right)$, possui uma boa capacidade para caracterização e delineamento de classes de uso e cobertura na porção sudoeste da Amazônia.

Mais recentemente, com a disponibilidade das imagens COSMO-SkyMed (COnstellation of small Satellites for Mediterranean basin Observation), de banda X, algumas aplicaçóes são relatadas: mapeamento de áreas agrícolas (Paloscia et al. 2012), de uso e cobertura da terra (Azevedo et al. 2012; Gomes et al. 2012) e estudos florestais (Weydahl e Eldhuset 2012). Há, contudo, um espaço importante de pesquisa no uso dessas imagens do COSMO-SkyMed, face à escassez de trabalhos em áreas tropicais.

Segundo Ulaby et al. (1986), Kurvonen e Hallikainen (1999) e Sarker et al. (2012), a agregação de informaçóes texturais às imagens SAR aumenta o potencial de discriminação das classes de uso e cobertura da terra. Nesse contexto, Kurvonen e Hallikainen (1999) analisaram os efeitos da combinação das imagens intensidade dos sensores ERS-1 (banda C) e JERS-1 (banda L) com os atributos de textura GLCM (Gray Level Co-occurrence Matrix), obtidos a partir das mesmas imagens, para a discriminação de classes de cobertura do solo e tipos florestais.

Nesse contexto, o presente trabalho tem como objetivo a análise da capacidade dos dados multitemporais do sensor COSMO-SkyMed, nas polarizaçóes HH-HV e VV-VH, no formato intensidade, e a avaliação da integração das informações texturais às imagens SAR, na identificação temática de uso e cobertura da terra no sul do estado do Amazonas (AM).

\section{MATERIAL E MÉTODOS}

\section{Área de Estudo}

A área de estudo localiza-se no município de Humaitá, sul do Estado do Amazonas, entre as coordenadas de 7o 23' - 7o 41' S e 63 03' e 63 24' W, perfazendo uma área de aproximadamente $1.007 \mathrm{~km}^{2}$ (Figura 1).

Está situada na sub-regiáo do Rio Madeira, sobre a Planície Amazônica (Martins et al. 2006), sendo composta pelas unidades pedológicas de argissolo, latossolo, cambissolo, gleissolo e plintossolo (IBGE, 2010a). De acordo com a classificação de Köppen, o clima da regiáo é do tipo Am (tropical úmido), caracterizado por temperatura média anual de $25^{\circ} \mathrm{C}$ e uma estação seca de pequena duraçáo, entre os meses de junho e agosto. Os elevados índices de precipitação na estação chuvosa compensam a estiagem, com valores entre 2050 a $2650 \mathrm{~mm}$ por ano, com elevada umidade do ar, variando entre 85 e 90\% (INMET, 2009).

A cobertura vegetal da área é formada por Floresta Ombrófila Aberta das Terras Baixas, com manchas de Savana (Cerrado) (IBGE 2010b). O domínio Savana, também chamado de Cerrado, aparece na regiáo de estudo na forma de contato (encraves), ocorrendo em meio à floresta, destacando duas formaçôes: Savana Arborizada e Savana GramíneoLenhosa, em acordo com a nomenclatura estabelecida por Veloso et al. (1991) e seguida no presente trabalho. A principal atividade econômica desenvolvida na regiáo é a agropecuária apresentando, segundo Becker (2005), expansáo de suas fronteiras no sul do Estado. Particularmente no município de Humaitá ocorrem, também, cultivos de grãos tais como arroz, milho, feijão e soja (Pinheiro e Rezende 2012).

\section{Materiais utilizados}

As imagens COSMO-SkyMed utilizadas neste trabalho foram adquiridas no modo StripMap, submodo Ping Pong, em passagens de dias consecutivos. A primeira imagem foi obtida pelo sensor em 01/09/2011, nas polarizaçôes HH e HV, e a segunda em 02/09/2011, nas polarizaçôes VV e VH. Os dados originais foram disponibilizados em formato Single Look Complex (SLC), cujas características estão contidas na Tabela 1. 


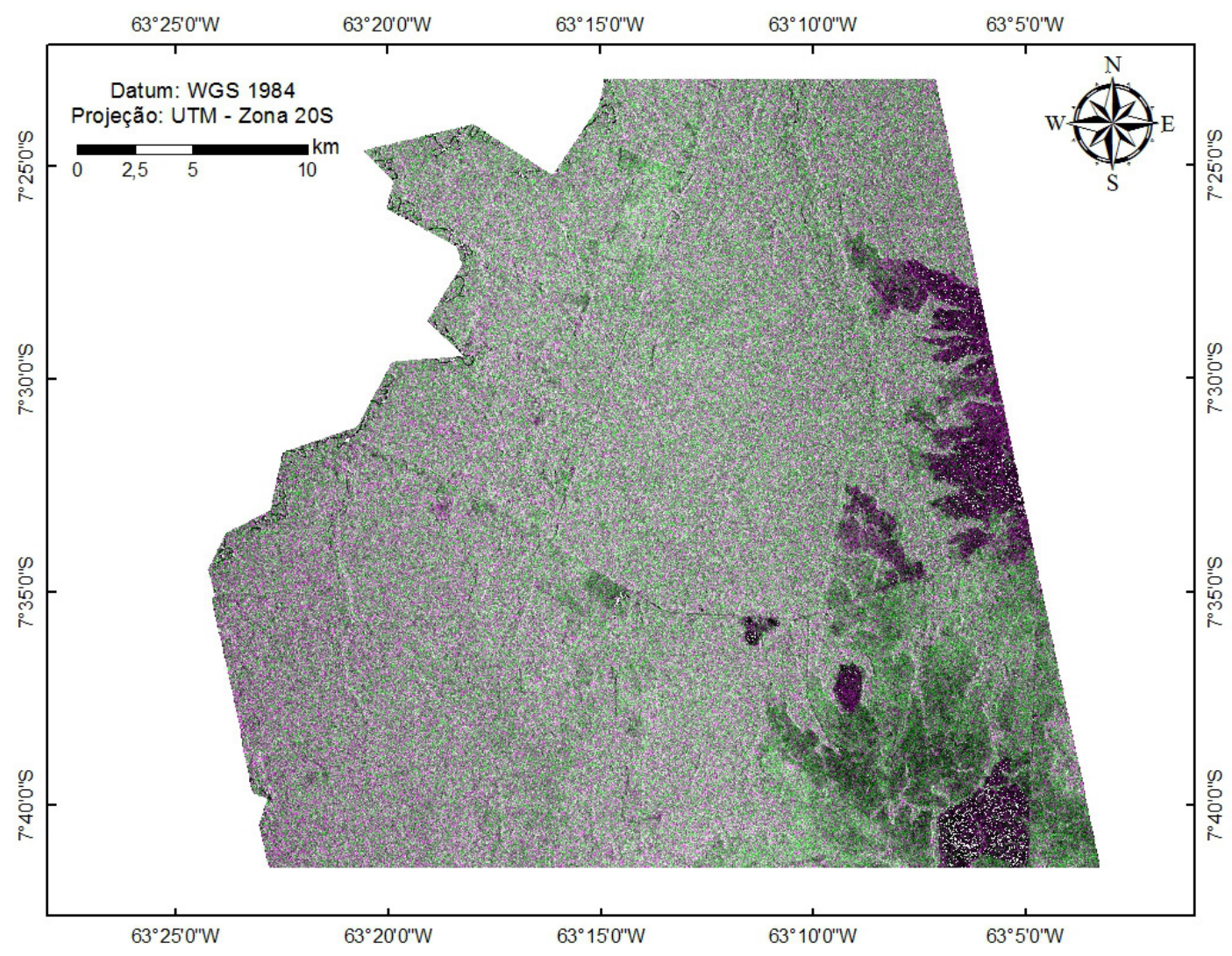

Figura 1 - Localização da área de estudo em composição colorida das imagens COSMO-SkyMed, sendo HH (em vermelho)-HV (verde)-HH (azul).

Como dados auxiliares foram utilizados informaçóes de campo, coletados em setembro de 2011, em período simultâneo ao imageamento do COSMO-SkyMed. Foram selecionados nesse trabalho de campo 210 pontos amostrais, representativos da paisagem, devidamente descritos, georreferenciados e fotografados, que subsidiaram a identificação temática de uso e cobertura da terra nas imagens obtidas pelo COSMOSkyMed. Adicionalmente foram utilizadas imagens ópticas (Thematic Mapper/Landsat-5: 05/07/2011 e 06/08/2011, e Linear Imaging Self-Scanner- LISS III/Indian Remote Sensing Satellite-IRS: 20/08/2011 e 13/09/2011), além da informação sobre a presença de focos de calor na regiáo de Humaitá, no período de 01/07/2011 a 02/09/2011, oriundos do projeto PROARCO/INPE (http://www.dpi.inpe.br/proarco/ bdqueimadas/), como subsídio à análise interpretativa das condições do uso e cobertura da terra no período investigado. Os dados de focos de calor foram utilizados como informaçáo complementar para a averiguaçáo de ocorrência de áreas de queimadas nas imagens multitemporais COSMO-SkyMed (coletadas em 01 e 02/09/2011).

Neste trabalho, foram consideradas seis classes temáticas: Floresta, abrangendo as áreas de Floresta Ombrófila Aberta e de Sucessão Secundária em estágios intermediários e avançado de regeneração; Floresta Aluvial, que ocorre ao longo dos cursos d'água ocupando terraços antigos das planícies quaternárias; Savana, incluindo os subgrupos GramíneoLenhosa e Arborizada, bem como aquelas em condiçôes antropizadas, decorrentes muitas vezes da remoçâo dos componentes florísticos naturais e substituição por práticas agrícolas (principalmente consorciadas ao cultivo de arroz e milho) e/ou de pecuária em determinado período sazonal; Reflorestamento, representado por área com plantação de Teca (Tectona grandis); Pasto, compreendendo áreas de Pasto Sujo (que corresponde às pastagens sem adequadas condiçôes de manejo ou recém abandonadas, com presença de babaçu (Attalea speciosa Mart. ex Spreng) em diferentes densidades de ocorrência ou mesmo espécies pioneiras típicas de elevada capacidade de disseminação no processo regenerativo natural e de Pasto Limpo, onde há o predomínio de vegetação graminóide adequada ao pastoreio, em geral formada por Brachiaria sp; Queimada, formada por açáo recente do fogo sobre a tipologia vegetal, localizada principalmente em áreas de Savana. 
Tabela 1 - Características das imagens COSMO-SkyMed no modo StripMap/ Ping Pong.

\begin{tabular}{lcc}
\hline & Imagem 1 & Imagem 2 \\
\hline Passagem & Ascendente & Ascendente \\
Data & $01 / 09 / 2011$ & $02 / 09 / 2011$ \\
Dimensão $(\mathrm{km})$ & $30 \times 40 \mathrm{~km}$ & $30 \times 40 \mathrm{~km}$ \\
Resolução $(\mathrm{m})$ & $15 \times 15$ & $15 \times 15$ \\
Polarizações & $\mathrm{HH} \mathrm{e} \mathrm{HV}$ & $\mathrm{VV} \mathrm{e} \mathrm{VH}$ \\
Ângulo de Incidência & $27,5^{\circ}$ & $27,5^{\circ}$ \\
\hline
\end{tabular}

Com o intuito de utilizar as imagens duais multitemporais como dados quad-pol, foram também analisadas as informaçôes de precipitaçáo acumulada, provenientes do Sistema Nacional de Dados Ambientais (SINDA/INPE - http://sinda.crn2.inpe. $\mathrm{br} / \mathrm{PCD}$ ), nos períodos que precederam ao imageamento, sobretudo nos dias 01 e 02 de setembro, correspondentes à aquisição das imagens no modo dual COSMO-SkyMed, visando avaliar o teor de umidade nos alvos.

\section{Processamento SAR}

No presente trabalho o processamento das imagens SAR incluiu quatro fases: pré-processamento, análise exploratória, classificação e validação, conforme demonstrado de forma sumarizada na Figura 2. A fase de pré-processamento constou de: (1) extração do Modelo Digital de Elevação (MDE); (2) processamento multilook; (3) correção de pixels com valores nulos, presentes nas polarizaçóes cruzadas HV e VH; (4) corregistro das imagens; (5) aplicação do filtro; (6) geocodificação; (7) análise das polarizaçôes cruzadas; (8) extração dos atributos texturais; (9) padronização das imagens intensidade e texturais.

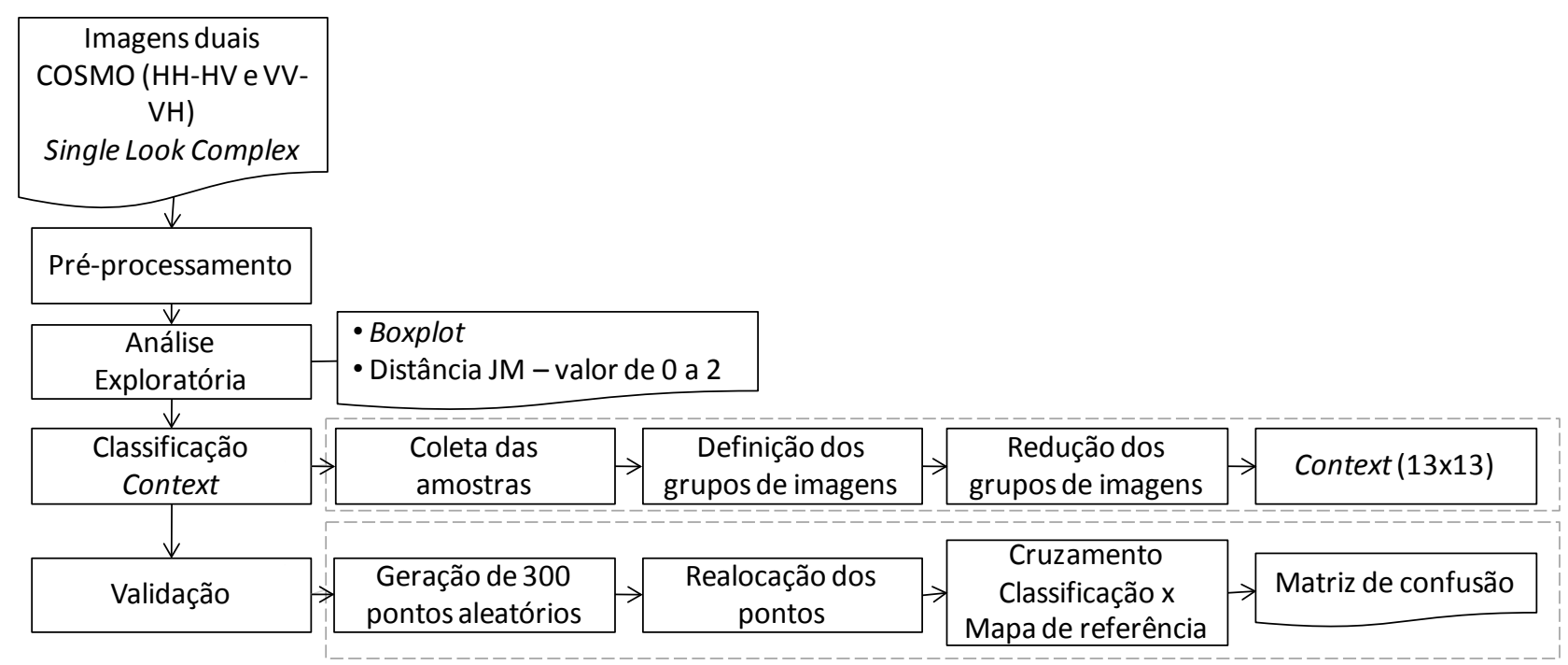

Figura 2 - Abordagem metodológica do processamento realizado nas imagens COSMO-SkyMed.
A partir das imagens no formato Single Look Complex (SLC), foi obtido o Modelo Digital de Elevação (MDE), a ser aplicado na etapa de geocodificação. Para tanto, utilizou-se como base o SRTM (Shuttle Radar Topography Mission), obtido no endereço eletrônico do Consortium for Spatial Information - CGIAR-CSI (http://srtm.csi.cgiar.org/), utilizando o software SARscape. As imagens SLCs, foram processadas no modo multilook, adotando dois looks na direção de range e 11 looks na direçáo de azimute, com vistas a reduzir o ruído speckle, além de ajustar as dimensóes das imagens. Dessa forma foram geradas imagens em slant range no formato intensidade para as polarizaçôes $\mathrm{HH}, \mathrm{HV}, \mathrm{VV}$ e $\mathrm{VH}$.

Analisando estas imagens, identificou-se a presença de pixels sem valores definidos (dummy pixels) nas imagens de polarizaçáo cruzada ( $\mathrm{HV}$ e $\mathrm{VH})$, que por sua vez foram substituídos pelo valor interpolado dos pixels vizinhos durante a etapa de geocodificação empregando o programa SARscape. O processamento posterior consistiu da aplicação do filtro Lee nas imagens multipolarizadas, com janela de tamanho 10x10 pixels. Apesar do processamento multilook ter sido aplicado previamente para a minimizaçáo do speckle, julgou-se necessário realizar uma redução mais aprofundada do ruído por meio da utilização do filtro. Ao final, as imagens foram geocodificadas a partir do MDE extraído anteriormente.

Com o intuito de se utilizar as imagens duais como dados quad-pol, foi realizada a análise das polarizaçóes cruzadas HV e $\mathrm{VH}$, considerando o princípio da reciprocidade, ou seja, que as intensidades dos sinais retroespalhados em $\mathrm{HV}$ e VH sejam iguais. Estas análises foram realizadas sobre o perfil do comportamento radiométrico dos sinais e dos valores de média e desvio padrão de polígonos das classes Floresta e Savana Gramíneo-Lenhosa nas imagens $\mathrm{HV}$ e VH, avaliando 


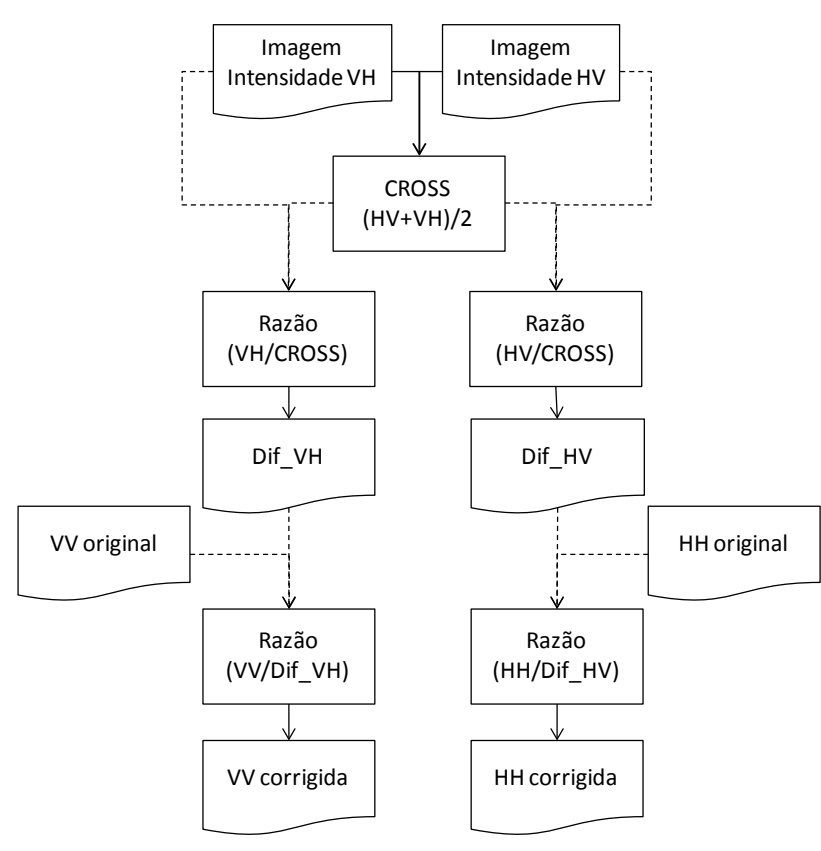

Figura 3 - Fluxograma da metodologia adotada para a correção das imagens adquiridas em datas diferentes.

a significância das diferenças encontradas, por meio do teste de hipótese de diferença de médias populacionais. Foram escolhidos dois polígonos representativos de cada classe, sendo considerado para a análise o valor médio entre eles. A utilização de apenas duas classes foi considerada suficiente uma vez que se trata de uma análise exploratória do sinal.

Tendo em vista a possibilidade de utilizaçáo do produto originário do imageamento multitemporal como dado quadpol, o procedimento metodológico a ser aplicado nessas imagens, para correção de diferenças na amplitude do sinal decorrente do multi-imageamento pode ser visto na Figura 3.

Incialmente foi realizada a média entre as imagens das polarizaçōes $\mathrm{HV}$ e $\mathrm{VH}$, obtendo-se dessa forma a imagem denominada "CROSS". Em seguida é produzida a imagemdiferença denominada "Dif_VH", resultante da razão entre as imagens VH e CROSS. Operação idêntica foi realizada para a imagem HV, obtendo-se então a imagem "Dif_HV". A partir desses produtos, realizam-se as correçôes das imagens co-polarizadas VV e HH por meio das razóes VV/Dif_VH e HH/Dif_HV, resultando nas imagens HH_cor e VV_cor. Dessa forma, as imagens corrigidas, são referenciadas ao longo deste artigo como HH, VV e CROSS (média entre HV e VH).

Os atributos texturais foram extraídos a partir das imagens $\mathrm{HH}, \mathrm{VV}$ e CROSS, isoladamente, adotando os parâmetros de textura GLCM (Gray Level Co-occurrence Matrix) (Haralick et al. 1973), utilizando janela com tamanho de $11 \times 11$ pixels. Dentre as texturas GLCM, foram consideradas: homogeneidade, contraste, dissimilaridade, média, desvio padrão, entropia e correlaçáo. Dessa forma, foram obtidas sete imagens texturais a partir da imagem $\mathrm{HH}$, sete de VV e sete de CROSS, totalizando 21 atributos texturais GLCM.

As imagens de textura apresentam distintas faixas de valores entre si e em relação às imagens intensidade. Com o intuito de evitar supervalorizar os atributos que apresentam valores maiores, durante o processo de classificação, optouse por padronizar todos os atributos, passando entấo a apresentarem valores entre zero e um, conforme metodologia aplicada por Soares et al. (1997). Para o cálculo dos valores máximos e mínimos foram considerados três desvios padrôes para as imagens intensidade e dois desvios padróes para os atributos de textura.

Para a definição dos grupos de imagens de textura, foi realizada uma análise exploratória, utilizando boxplot e distância Jeffries-Matusita (JM) de cada atributo textural, com o objetivo de avaliar suas capacidades de separabilidade para as classes temáticas. Para a elaboração dos boxplot, foram utilizados os valores médios de intensidade de cada polígono amostrado para cada tema.

A classificação Context, desenvolvida por Gong e Howarth (1992) e implementada no software PCI Geomatics, baseiase na frequência de ocorrência dos níveis de cinza dentro de uma determinada janela de pixels. Essa metodologia requer uma fase de treinamento, que consiste na coleta de amostras para subsidiar a classificação. Nesta fase foram coletados 105 polígonos de Floresta (171.475 pixels), 23 de Floresta Aluvial (2.085 pixels), 26 de Savana (9.776 pixels), um de Reflorestamento (1.032 pixels), 19 de Pasto (10.002 pixels) e 26 de Queimada (20.644 pixels). A classe Reflorestamento ocorre somente em uma pequena regiáo na área de estudo sendo, portanto, possível apenas a coleta de um polígono de amostragem.

Inicialmente, essa classificação necessita da redução dos grupos de imagens, gerando uma imagem de saída cujos níveis de cinza variam de 0 a 50 . Para tanto, foram definidos os grupos de imagens, que incluem as imagens intensidade e texturais. Em seguida, esses grupos foram reduzidos, gerando as imagens que subsidiaram as classificaçôes. Para a definição do tamanho de janela a ser utilizada na classificação, foi realizada uma avaliação utilizando janelas com dimensôes ímpares de $5 \times 5$ até $21 \times 21$ pixels. O melhor resultado foi alcançado pela janela de tamanho $13 \times 13$ pixels.

Como subsídio ao processo de validação das classificaçóes, foi elaborado um mapa referência a partir da imagem Landsat 5/TM, órbita/ponto 232/65, de 06 de agosto de 2011, acrescida das informaçôes de campo, dos focos de calor entre 01 de julho e 01 de setembro de 2011, além das imagens Landsat 5/TM de 05 de julho de 2011, IRS/LISS3, órbita/ponto 312/81, de 20 de agosto e 13 de setembro de 
Tabela 2 - Valores de média $(\mu)$ e desvio padrão $(\sigma)$ das classes Floresta e Savana Gramíneo-Lenhosa, nas polarizações VH e HV.

\begin{tabular}{|c|c|c|c|c|c|c|}
\hline \multirow[b]{2}{*}{ Classe } & \multirow[b]{2}{*}{$\mathrm{N}^{0}$ pixels } & \multicolumn{2}{|c|}{ VH } & \multicolumn{2}{|c|}{ HV } & \multirow{2}{*}{$\begin{array}{c}\mu \mathrm{VH}-\mu \mathrm{HV} \\
\text { Valor } \mathrm{Z}\end{array}$} \\
\hline & & $\mu$ & $\sigma$ & $\mu$ & $\sigma$ & \\
\hline \multirow{2}{*}{ Floresta } & 12.460 & 721,20 & 252,52 & 495,49 & 168,95 & \\
\hline & 24.444 & 803,09 & 266,24 & 569,10 & 182,26 & \\
\hline Média & 36.904 & 762,14 & 259,38 & 532,29 & 175,60 & 140,90 \\
\hline \multirow{2}{*}{ Savana Gramíneo-Lenhosa } & 315 & 628,28 & 225,73 & 413,34 & 142,96 & \\
\hline & 243 & 605,48 & 216,09 & 404,71 & 124,35 & \\
\hline Média & 4.471 & 616,88 & 220,91 & 409,02 & 133,65 & 19,00 \\
\hline
\end{tabular}

2011 além da própria imagem COSMO-SkyMed de 01 de setembro de 2011.

A fase de validação foi realizada no software ArcGis 9, com as imagens classificadas sendo exportadas do ambiente PCI para o ArcGis. Foram criados 300 pontos amostrais aleatoriamente e posteriormente realizou-se a análise da localização dos pontos e realocação dos mesmos, com base no mapa referência e com apoio do conhecimento de campo, com o intuito de que todas as classes fossem contempladas com o maior número de pontos possível. Posteriormente foi realizado o cruzamento entre os pontos de validação e as imagens classificadas, permitindo, dessa forma, a elaboraçáo das matrizes de confusão e o cálculo do índice Kappa estimado.

\section{RESULTADOS}

A partir dos resultados da análise das polarizaçóes cruzadas, verificou-se que os valores de média e desvio padrão do sinal retroespalhado na polarização VH das amostras de Floresta e Savana Gramíneo-Lenhosa são estatisticamente maiores em relação aos de HV (Tabela 2). Porém, ao comparar a variação dos valores de média e desvio padrão entre as classes, nota-se o mesmo comportamento do sinal retroespalhado em ambas as polarizaçôes. Assim, pode ser verificado que o valor da média da classe Floresta é maior do que o de Savana GramíneoLenhosa, tanto na polarização VH como em HV. Essa relação no comportamento radiométrico do sinal entre as polarizaçóes também pode ser verificado através do perfil radiométrico da linha 1.671 traçado nas imagens $\mathrm{HV}$ e VH (Figura 4).

Por sua vez, considerando os resultados obtidos na análise exploratória dos atributos texturais, foi possível definir oito grupos de imagens, sobre os quais foram realizadas classificações expeditas, para avaliar aquele de melhor desempenho temático. $\mathrm{O}$ grupo de imagens texturais formado pelos atributos Homogeneidade e Média de HH; Contraste, Dissimilaridade, Média e Desvio Padrão de CROSS; e Dissimilaridade, Média e Desvio Padráo de VV, apresentou valor médio de distância JM de 1,68 e alcançou o melhor resultado na classificação (acurácia total $=64,33 \%$ e Kappa $a=$ $0,553)$, comparado aos demais possíveis grupos de formação. Portanto, esse grupo textural foi utilizado nas classificaçóes, juntamente com as imagens intensidade.
Para a etapa metodológica de validação de todas as classificaçóes possíveis, foi utilizado o mapa referência (Figura 5). As classificaçóes contextuais foram realizadas utilizando sete grupos de imagens, que incluíram as imagens intensidade nas três polarizaçóes e o grupo de textura mencionado anteriormente, de forma isolada e agrupada, considerando as seis classes temáticas de interesse. A partir das validaçôes das classificaçôes foram obtidos os valores de acurácia total e índice Kappa (Tabela 3).

Observa-se que o grupo $\mathrm{HH}+\mathrm{CROSS}+\mathrm{VV}$ apresentou melhor desempenho temático, com $66 \%$ de acurácia total e valor Kappa de 0,554 (concordância moderada), quando comparado aos demais grupos, inclusive aqueles nos quais estấo inseridas as medidas texturais. Não obstante, estatisticamente os resultados obtidos nas demais classificaçóes não apresentam diferença significativa, ao nível de 5\% de significância, quando comparado ao melhor resultado. A matriz de confusão originada da classificação $\mathrm{HH}+\mathrm{CROSS}+\mathrm{VV}$ pode ser visualizada na Tabela 4, enquanto que o resultado dessa classificação é mostrado na Figura 6.

\section{DISCUSSÃO}

Considerando os resultados obtidos pela análise do comportamento radiométrico das imagens $\mathrm{HV}$ e $\mathrm{VH}$, e a inexistência de variaçóes significativas no índice pluviométrico para a regiáo entre as datas dos dois imageamentos, podemos aceitar como premissa que a diferença na intensidade do sinal é consequência exclusivamente de uma possível diferença nas potências de emissão ou mesmo variação no ganho das antenas dos radares utilizados nas duas passagens. Decorrente do conhecimento obtido em campo, pode-se afirmar que o comportamento radiométrico dos sinais indica não haver alteraçôes na condição fisionômico-estrutural e na capacidade fotossintética da vegetaçấo presente na paisagem, entre as duas datas das imagens investigadas. Essas análises indicaram a possibilidade de utilizar as imagens duais de forma conjunta, ou seja, como um dado quad-pol. Para tanto, foi realizada a correção das imagens, conforme indicado na Figura 3, de modo a retirar as diferenças de efeitos radiométricos supracitadas, consideradas estatisticamente significativas, entre as imagens adquiridas em datas diferentes. 


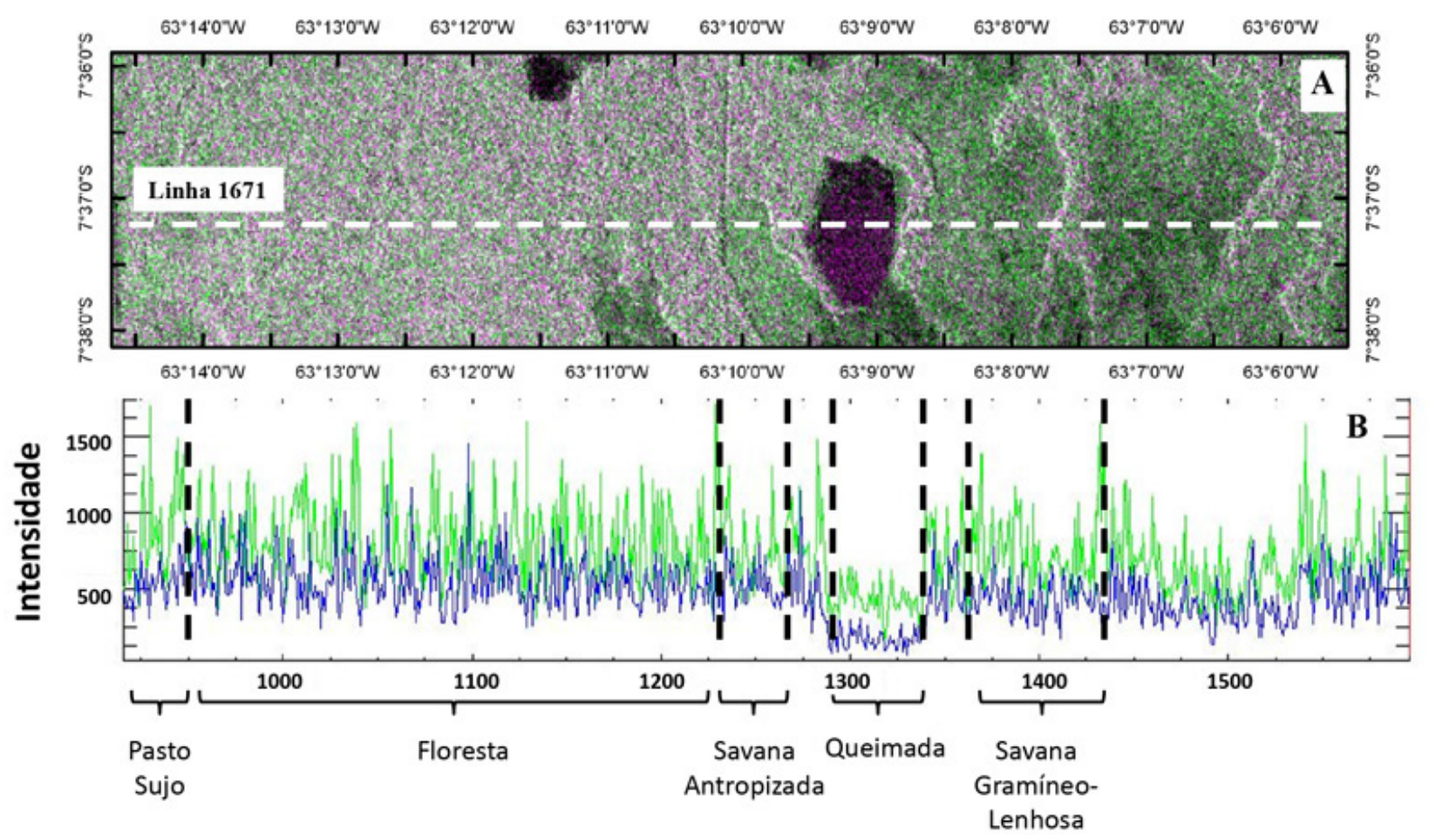

Figura 4 - Perfil da linha 1671 (demarcação tracejada) nas imagens de polarizações HV e VH do COSMO-SkyMed (A), e o respectivo comportamento radiométrico dos sinais para as classes temáticas presentes na cena, sendo o sinal de VH representado pela linha verde e o sinal HV pela linha azul (B).

A partir da análise de matriz de confusão da melhor classificaçáo obtida $(\mathrm{HH}+\mathrm{CROSS}+\mathrm{VV})$ é possível notar a existência de similaridade no espaço de atributos radiométricos das imagens COSMO-SkyMed, entre as classes Reflorestamento e Floresta, decorrente dos mecanismos de espalhamento superficial e, talvez, volumétrico na banda $\mathrm{X}$ perante a estrutura de dossel dessas tipologias citadas. A Floresta Aluvial também apresentou algumas áreas classificadas como Floresta, indicando haver certa similaridade na configuração do espaço de atributos radiométricos na imagem COSMO-SkyMed.

Ainda decorrente dessa matriz de confusão, observa-se também que a classe Queimada apresentou certa similaridade radiométrica com Savana, fato causado devido às regióes de queimadas serem constituídas, em sua maior parte, por áreas de Savana Arborizada. Em geral, a queima ocorre no estrato herbáceo, atingindo alguns indivíduos arbustivo-arbóreos, cuja estrutura fisionômica permanece. A banda X, por apresentar maior interação com o topo da cobertura vegetal, sem alcance de penetração no alvo, detecta a sua estrutura que mesmo após a queima pode permanecer conservada, gerando assim uma confusão espectral entre essas classes.

Outro caso que apresenta alguma similaridade de configuraçôes radiométricas é o de áreas de pastagem. Essas são compostas geralmente por um tapete graminóide, com indivíduos arbustivos esparsos, decorrente do processo regenerativo natural. Mas foi constatada uma elevada densidade de palmeiras (em especial babaçu) em diferentes

Tabela 3 - Valores de acurácia, Kappa e variância do Kappa das classificações.

\begin{tabular}{lccc}
\hline Grupos & Acurácia (\%) & Kappa & Variância do Kappa \\
\hline HH + CROSS & 64,67 & 0,553 & 0,001190417 \\
VV + CROSS & 61,67 & 0,519 & 0,001174297 \\
HH + CROSS +VV & 65,67 & 0,554 & 0,001240121 \\
Textura & 64,33 & 0,553 & 0,001122994 \\
HH + CROSS + VV + Textura & 64,67 & 0,559 & 0,001156269 \\
HH + CROSS + Textura & 59,67 & 0,498 & 0,001247117 \\
VV + CROSS + Textura & 62,00 & 0,522 & 0,001186992 \\
\hline
\end{tabular}




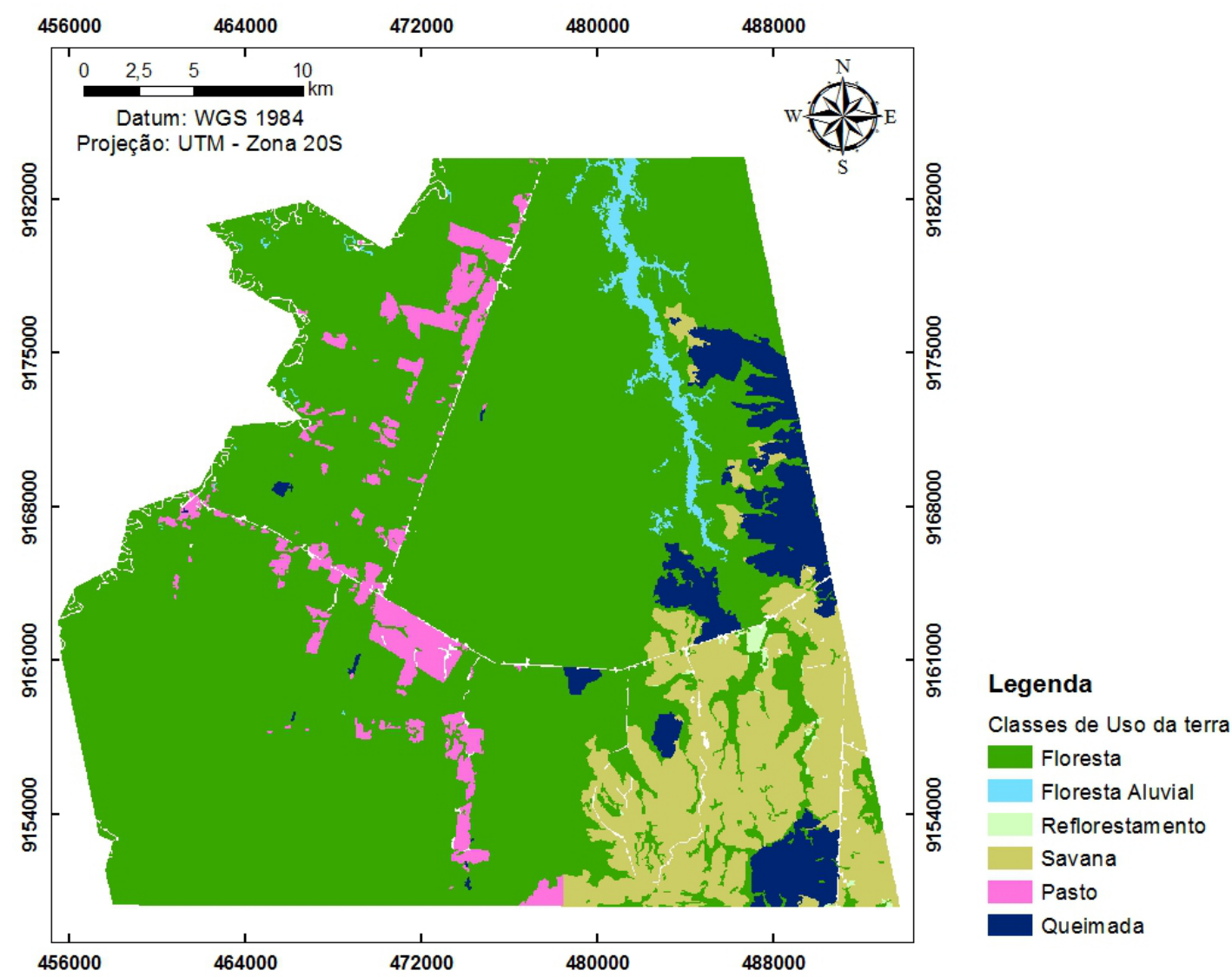

Figura 5 - Mapa referência da área de estudo utilizada na etapa de validação.

graus de crescimento vegetativo, razão da ausência de práticas de manejo adequadas às condiçốes de pastoreio. Assim, a variação estrutural dessas áreas de pastagem passa a ocupar um espaço de atributos radiométricos similar, na banda X, àquele da classe Savana Gramíneo-Lenhosa, e até mesmo como alvos de estrutura florestal. Stolz e Mauser (1995) também apontaram dificuldade em discriminar as classes Pasto e Floresta ao realizar a classificaçáo de imagens amplitude multitemporais do sensor em banda $\mathrm{X}$, na polarização $\mathrm{VV}$.
Segundo os autores, essa diferenciação é possível com a utilização da banda L, que apresenta maior penetrabilidade. Acrescente-se ainda que, além das atividades de pastoreio serem realizadas em áreas onde a floresta foi derrubada e convertida em pasto, é comum nessa região a atividade de pecuária extensiva desenvolvida no próprio domínio savânico, o que torna também difícil uma discriminação específica com os dados sensoriados em banda X, entre o tema Savana e Pasto.

Tabela 4 - Matriz de confusão da classificação realizada com o grupo de imagens intensidade HH+CROSS+VV.

\begin{tabular}{|c|c|c|c|c|c|c|c|}
\hline \multicolumn{8}{|c|}{ MAPA REFERÊNCIA (\%) } \\
\hline \multirow{7}{*}{ 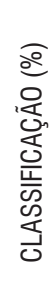 } & Classes & Floresta & FI. Aluvial & Reflorest & Savana & Pasto & Queimada \\
\hline & Floresta & 82,8 & 22,7 & 77,8 & 2,8 & 35,6 & 2,5 \\
\hline & Fl. Aluvial & 7,1 & 72,7 & 0,0 & 11,3 & 15,3 & 0,0 \\
\hline & Reflorest & 3,0 & 0,0 & 22,2 & 0,0 & 5,1 & 0,0 \\
\hline & Savana & 3,0 & 0,0 & 0,0 & 70,4 & 16,9 & 20,0 \\
\hline & Pasto & 3,0 & 4,5 & 0,0 & 7,0 & 27,1 & 0,0 \\
\hline & Queimada & 1,0 & 0,0 & 0,0 & 8,5 & 0,0 & 77,5 \\
\hline
\end{tabular}




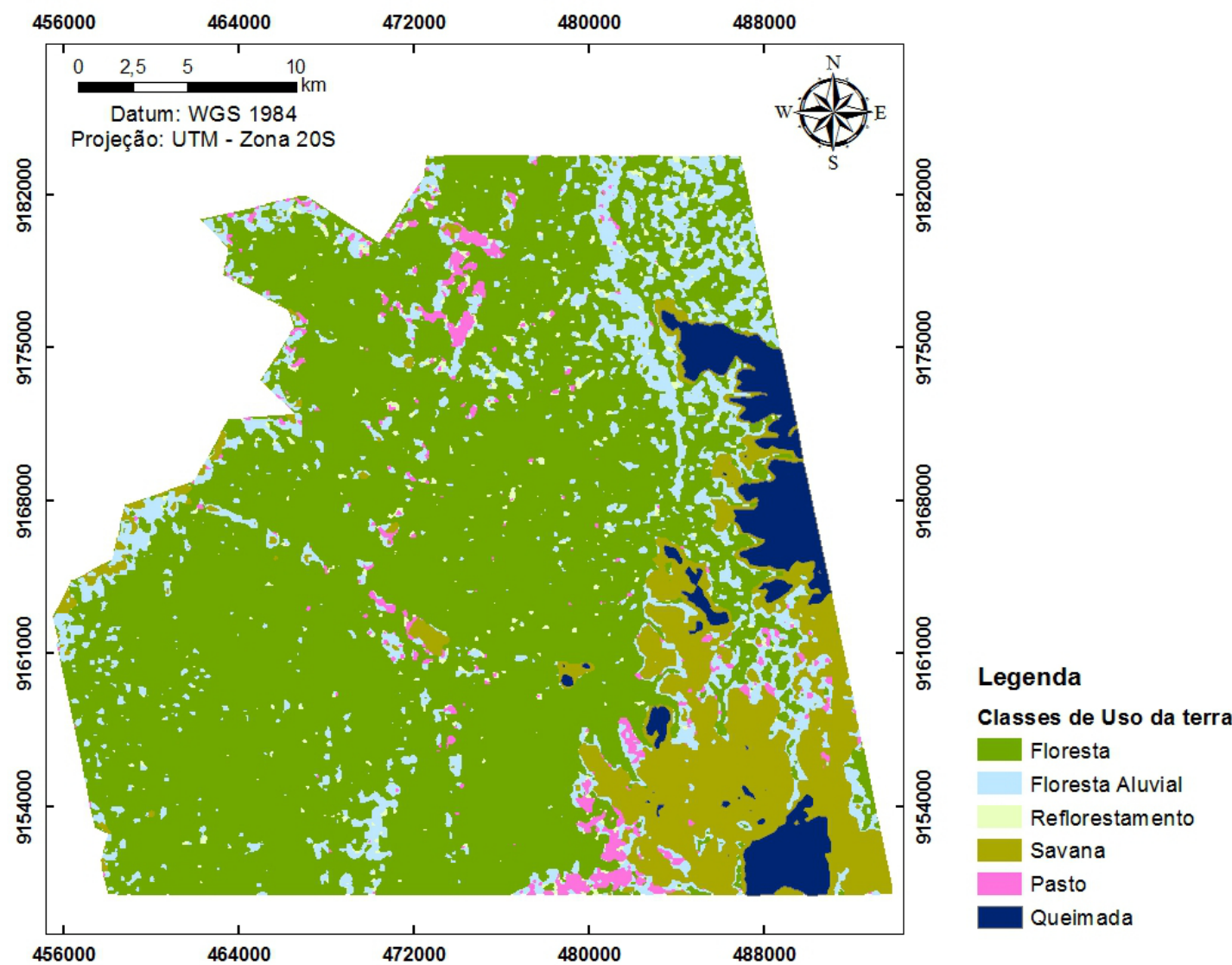

Figura 6 - Mapa da classificação Context utilizando o grupo de imagens intensidade HH+CROSS+VV.

O resultado deste trabalho diverge do exposto em estudos realizados por Van Der Sanden e Hoekman (1999) e Kurvonen e Hallikainen (1999), que sugerem a utilização dos atributos texturais com o intuito de melhorar significativamente a classificação de cobertura e uso da terra. É importante registrar que tais pesquisas citadas foram desenvolvidas com o emprego de classificador estatístico pontual, como o MAXVER, cujos algoritmos não são adaptados ao tipo de distribuição estatística e da presença de speckle que os dados SAR apresentam. Assim, ao utilizar um classificador contextual, mais adaptado às características radiométricas dos dados SAR, as classificaçóes utilizando somente imagens intensidade indicam resultados superiores em termos absolutos de desempenho temáticos, mas estatisticamente similares àqueles obtidos com a agregação de informação textural.

\section{CONCLUSÕES}

A avaliação das imagens duais HH-HV e VV-VH, oriundas de imageamento multitemporal, do satélite COSMO-SkyMed, no sub-modo Ping Pong, mostra através do comportamento radiométrico destas polarizaçôes a possibilidade de uso como imagens quad-pol intensidade. Isto fica confirmado pelo melhor desempenho temático obtido pela classificaçáa realizada a partir do grupo de imagens intensidade formado pelas polarizaçóes $\mathrm{HH}, \mathrm{VV}$ e CROSS (média entre $\mathrm{HV}$ e $\mathrm{VH}$ ). Entretanto este desempenho não apresenta diferença estatística significativa ao nível de 5\% de significância, se comparado com aqueles obtidos pelos grupos formados pelas imagens $\mathrm{HH}+\mathrm{CROSS}$ e $\mathrm{VV}+\mathrm{CROSS}$ e tampouco, com a inclusão da diversidade de atributos texturais investigados.

$\mathrm{Na}$ expectativa de aprimorar o mapeamento e monitoramento da paisagem amazônica, a partir da utilização de dados de banda X, sugere-se também investigar o uso da coerência interferométrica derivada dos dados multitemporais do COSMO-SkyMed, sub-modo HImage.

\section{AGRADECIMENTOS}

Agradecemos à FAPESP (Fundação de Amparo à Pesquisa do Estado de São Paulo -Projeto no. 2011/05917. 4) pelo suporte financeiro, à CAPES (Coordenaçáo de Aperfeiçoamento de Pessoal de Nível Superior) pela bolsa de pesquisa de pós-graduação, ao $\mathrm{CNPq}$ (Conselho Nacional de Desenvolvimento Científico e Tecnológico) pela bolsa de produtividade em pesquisa e à Telespazio pela disponibilizaçáo das imagens COSMO-SkyMed. 


\section{BIBLIOGRAFIA CITADA}

Asner, G. P. 2001. Cloud cover in Landsat observations of the Brazilian Amazon. International Journal of Remote Sensing, 22: 3855-3862.

Azevedo, A. R.; Gama, F. F.; Santos, J. R.; Mura, J. C.; Graça, P. L. A. 2012. Análise da integração de atributos de textura em imagens SAR Cosmo-Skymed como subsídio à classificação de uso e cobertura da terra. Anais... X Seminário de Atualização em Sensoriamento Remoto e Sistemas de Informaçóes Geográficas Aplicados à Engenharia Florestal. Curitiba. Brasil. Curitiba: IEP. p. 221-228. ISSN 2178-8634. CD-ROM.

Balzter, H. 2001. Forest mapping and monitoring with interferometric synthetic aperture radar (InSAR). Progress in Physical Geography, 25: 159-177.

Becker, B. K. 2005. Geopolítica da Amazônia. Estudos Avançados, 19: 71-86.

Garcia, C. E.; Santos, J. R.; Mura, J. C.; Kux, H. J. H. 2012. Análise do potencial de imagem TerraSAR-X para mapeamento temático no sudoeste da Amazônia brasileira. Acta Amazônica, 42: 205-214.

Gomes, A. R.; Sadeck, L. W. R.; Brandão, W. S. Mapping land use cover in Amazon region with COSMO SkyMed data. Proceedings... International Geoscience and Remote Sensing Symposium. Munich. Germany. Munich: IEEE Press. p. 6539-6540. ISBN 978-1-4673-1160-1.

Gonçalves, F. G.; Santos, J. R.; Treuhaft, R. N. 2011. Stem volume of tropical forests from polarimetric radar. International Journal of Remote Sensing, 32: 503-522.

Gong, P.; Howarth, P. J. 1992. Frequency-based contextual classification and gray-level vector reduction for land-use identification. Photogrammetric Engineering \& Remote Sensing, 58: 423-437.

Graça, P. M. L. A.; Maldonado, F. D.; Fearnside, P. M. 2007. Detecção de desmatamento em novas áreas de expansão agropecuária no sul do Amazonas utilizando imagens CBERS-2. Anais... XIII Simpósio Brasileiro de Sensoriamento Remoto. Florianópolis. Brasil. São José dos Campos: INPE. p. 917-924. ISBN 978-85-17-00031-7.

Haralick, R. M.; Shanmugam, K.; Dinstein, I. 1973. Textural features for image classification. IEEE Transactions on Systems, Man and Cybernetics, SMC-3: 610-621.

Hoekman, D. H.; Quiñones, M. J. 2000. Land cover type and biomass classification using AirSAR data for evaluation of monitoring scenarios in the Colombian Amazon. IEEE Transactions on Geoscience and Remote Sensing, 38: 685-696.

IBGE. 2010a. Estado do Amazonas: Pedologia - Mapa Exploratório de Solos. Rio de Janeiro: IBGE. 1 Mapa.
Escala 1:250.000. Disponível em: < http://www.ibge.gov. br/home/geociencias/default_prod.shtm\#GEOG $>$. Acesso em: 21/01/2012.

. 2010b. Estado do Amazonas: Vegetação. Rio de Janeiro. 1 Mapa. Escala 1:250.000. Disponível em: < http://www.ibge.gov.br/home/geociencias/default_prod. shtm\#GEOG>. Acesso em: 21/01/2012.

INMET. 2009. Normais climatológicas do Brasil 1961-1990. Brasília: INMET. Disponível em: <http://www.inmet. gov.br/html/clima/mapas/? mapa=prec $>$. Acesso em: 26/01/2012.

Kasischke, E. S.; Melack, J. M.; Dobson, M. C. 1997. The use of imaging radars for ecological applications: A review. Remote Sensing of Environment, 59: 141-156.

Kurvonen, L.; Hallikainen, M. T. 1999. Textural information of multitemporal ERS-1 and JERS-1 SAR images with applications to land and forest type classification in boreal zone. IEEE Transactions on Geoscience and Remote Sensing, 37: 680-689.

Le Toan, T.; Quegan, S.; Davidson, M.W.J.; Balzter, H.; Paillou, P.; Papathanassiou, K.; Plummer, S.; Rocca, F; Saatchi, S.; Shugart, H.; Ulander, L. 2011. The BIOMASS mission: Mapping global forest biomass to better understand the terrestrial carbon cycle. Remote Sensing of Environment, 115: 2850-2860.

Martins, G. C.; Ferreira, M. M.; Curi, N.; Vitorino, A. C. T.; Silva, M. L. N. 2006. Campos nativos e matas adjacentes da região de Humaitá (AM): Atributos diferenciais dos solos. Ciência e Agrotecnologia, 30: 221-227.

Paloscia, S.; Pettinato, S.; Santi, E. 2012. Combining L and X band SAR data for estimating biomass and soil moisture of agricultural fields. European Journal of Remote Sensing, 45: 99-109.

Pinheiro, E. S.; Rezende, M. G. G. 2012. Análise do desflorestamento no sul do Amazonas. ACTA Geográfica, 6: 175-192.

Saatchi, S.; Marlier, M.; Chazdon, R. L.; Clark, D. B.; Russell, A. E. 2011. Impact of spatial variability of tropical forest structure on radar estimation of aboveground biomass. Remote Sensing of Environment, 115: 2836-2849.

Santos, J. R.; Freitas, C. C.; Araujo, L. S.; Dutra, L. V., Mura, J. C.; Gama, F. F.; Soler, L. S.; Sant'anna, S. J. S. 2003. Airborne P-band SAR applied to the aboveground biomass studies in the Brazilian tropical rainforest. Remote Sensing of Environment, 87: 482-493.

Sarker, M. L. R.; Nichol, J.; Ahmad, B.; Busu, I.; Rahman, A. A. 2012. Potential of texture measurements of two-date dual polarization PALSAR data for the improvement of 


\section{ACTA}

forest biomass estimation. ISPRS Journal of Photogrammetry and Remote Sensing, 69: 146-166.

Soares, J. V.; Rennó, C. D.; Formaggio, A. R.; Yanasse, C. C. F.; Frery, A. C. 1997. An investigation of the selection of texture features for crop discrimination using sar imagery. Remote Sensing of Environment, 59: 234-247.

Stolz, R.; Mauser, 1995. W. First evaluations of Shuttle SIR-C and X-SAR data for landcover classifications. Proceedings... International Geoscience and Remote Sensing Symposium. Firenze. Italy. Firenzi: IEEE Press. p. 1058-1060. ISBN 0-7803-2567-2.

Ulaby, F. T.; Kouyate, F.; Brisco, B.; Williams, T. H. L. 1986. Textural information in SAR images. IEEE Transactions on Geoscience and Remote Sensing, GE-24: 235-245.

Van Der Sanden, J. J.; Hoekman, D. H. 1999. Potential of airborne radar to support the assessment of land cover in a tropical rain forest environment. Remote Sensing of Environment, 68: 26-40.
Veloso, H. P.; Rangel-Filho, A. L. R.; Lima, J. C. A. 1991. Classificação da vegetação brasileira, adaptada a um sistema universal. Rio de Janeiro: IBGE, 124p. ISBN: 85-2400384-7.

Yanasse, C. C. F.; Sant'anna, S. J. S.; Frery, A. C.; Rennó , C. D.; Soares,J. V.; Luckman, A. J. 1997. Exploratory study of the relationship between tropical forest regeneration stages and SIR-C L and C data. Remote Sensing of Environment, 59: 180-190.

Weydahl, D. J.; Eldhuset, K. 2012. Combining polarimetric and coherence SAR images for forest type discrimination. Proceedings... IX European Conference on Synthetic Aperture Radar. Nuremberg. Germany. Nuremberg: IEEE Press, p. 235-238.

Recebido em 31/01/2013

Aceito em 05/06/2013 
